This document is confidential and is proprietary to the American Chemical Society and its authors. Do not copy or disclose without written permission. If you have received this item in error, notify the sender and delete all copies.

\title{
Micropipette-tip based immunoassay with electrochemical detection of anti-tissue transglutaminase to diagnose celiac disease using staples and a paper-based platform
}

\begin{tabular}{|c|c|}
\hline Journal: & ACS Sensors \\
\hline Manuscript ID & se-2019-01096y.R2 \\
\hline Manuscript Type: & Article \\
\hline $\begin{array}{r}\text { Date Submitted by the } \\
\text { Author: }\end{array}$ & $\mathrm{n} / \mathrm{a}$ \\
\hline Complete List of Authors: & $\begin{array}{l}\text { González López, Andrea; Universidad de Oviedo } \\
\text { Blanco-López, Maria Carmen; Universidad de Oviedo, Química Física y } \\
\text { Analítica } \\
\text { Fernández-Abedul, M. Teresa; Universidad de Oviedo, Química Física y } \\
\text { Analítica }\end{array}$ \\
\hline
\end{tabular}

\section{SCHOLARONE ${ }^{m}$ Manuscripts}




\begin{abstract}
In this work, 1-200 $\mu \mathrm{L}$ polypropylene micropipette tips were used as the platform for performing immunoassays after converting their inner surface on a capture zone for the analyte of interest. We have used a micropipette-tip immunoelectroanalytical platform for the detection of anti-tissue transglutaminase (IgA), the main biomarker for the celiac disease. Modification of the tip wall with poly-L-lysine allowed adsorption of tissue transglutaminase (tTG), which will capture later anti-tTG (IgA) antibodies developed in celiac people. A sandwich-type format was followed, incubating simultaneously the analyte and the detection antibody, labeled with horseradish peroxidase. With this new application for an extremely common lab material, we can perform quantitative analysis by dispensing the liquid into a low-cost and miniaturized staple-based paper electrochemical platform. The analytical signal was the reduction of the enzymatically-oxidized substrate, recorded chronoamperometrically (i-t curve). The intensity of the current obtained at a fixed time after application of the cathodic potential followed a linear relationship with anti-tTG (IgA) concentration. The relative standard deviation (RSD) obtained for immunoassays performed in different tips, indicates the adequate precision of this new methodology, very promising for decentralized analysis. Negative and positive controls produced results that were in accordance with those obtained with spectrophotometric ELISAs.
\end{abstract}

Reducing the size of the analytical devices, the complexity of the procedures, the analysis time and the cost of assays is becoming urgently required in many and different fields, where yet sensitive and selective determinations are needed. In this context, early diagnosis is essential to achieve a successful disease treatment. On this basis, rapid, robust, reliable and lowcost devices are being increasingly demanded, especially in developing countries and areas with limited resources. ${ }^{1}$ Electrochemical immunoassays take advantage of both, the specificity of antibodies and the sensitivity of a physicochemical transducer that transforms a biological response into a readable signal.

Amperometric detection, based on the measurement of an intensity of current, only requires: i) an appropriate conductive surface to act as working electrode, ii) an adequate reference electrode for applying potentials precisely, iii) an auxiliary electrode to close the circuit, in case a large current is measured, iv) an electrolyte to connect ionically all the electrodes and v) connection between the electrodes and the potentiostat, equipment that is becoming also miniaturized. ${ }^{2}$ All this can be accomplished using low-cost substrates, such as paper. This material has been used for long in analysis, but after pioneer works of Whitesides' group on $\mu \mathrm{PADs}^{3}$ and Henry's group on $\mu \mathrm{PEDs}^{4}$ it has gained increasing interest. With paper-based approaches, electrochemical cells have gained in flexibility (when compared with traditional glass cells), miniaturization (a paper-based cell with electrodes could be designed in an area lower than $1 \mathrm{~cm}^{2}$ ), versatility (based on papers with very different characteristics, $\left.{ }^{5,6}\right)$, reduction of electrolyte volume when compared with conventional cells that used commonly 10$20 \mu \mathrm{L}$, reduction of costs (paper is a cheap abundant material), portability (due to their reduced size with integrated electrodes), disposability (although many low-cost connectors can be reused) and integration with other steps of the analytical process through e.g., 3D structures. ${ }^{8}$ Nowadays, there are applications to determine analytes with clinical relevance, such as glucose in whole blood or relevant analytes in urine and saliva, ${ }^{9,10}$ but also other with interest in agro-food ${ }^{11}$ or environmental sectors. ${ }^{12}$ Apart from these advantages, electrochemical detection does not need transparent or colorless samples, has low power requirements and does not use expensive or complicate instrumentation.

The most common electrodes are those based on carbon ink ${ }^{13}$ although metal films and wires ${ }^{14}$ can also be employed. In an innovative approach, conductive mass-produced elements of common use have been modified with carbon ink for use as electrodes. This is the case of stainless-steel pins ${ }^{15,16}$ or staples ${ }^{17}$, out-of-box applications of materials commonly employed for a different purpose.

In this work, we propose the use of micropipette tips for performing immunoassays after converting their inner surface to a capture zone for the analyte. The electrochemical detection is later performed dropping the solution containing a detectable species on a staple paper-based cell. ${ }^{17}$ This is a totally new application for the tips, with a function that differs considerably from the original one. Micropipettes have been used for many years in routine laboratories for dispensing liquids, since they allow to work precisely with low sample volumes, they are light and ergonomic and thus, easy to handle and they use low-cost mass-produced tips, that can be discarded after use, avoiding cross contamination between samples. Therefore, micropipettes 
are among the most common volumetric materials found in laboratories of many different fields.

This is not the first time that micropipette tips have been used for functions different from dispensing liquids. They have been also used for facilitating sample preparation (packed with commercial resins ${ }^{18,19}$ ) and for performing electrochemical measurements, as containers of electrodes and electrolyte ${ }^{20,21}$ or just as a part of the electrode body (commonly working ${ }^{22}$ or reference ${ }^{23}$ electrodes). The tip can be used as a miniaturized column that can be filled with commercial resins for purification purposes. ${ }^{24}$ The ZipTip ${ }^{\circledR}$ is a special micropipette tip ${ }^{25}$ with a bed of chromatography media (C18, C4 or strong cationic) fixed at its end, intended for purifying and concentrating protein, peptide or oligonucleotide samples by solid-phase extraction prior to analysis (usually mass spectrometry). Specific purifications can be made by modifying the micropipette tip with molecularly imprinted monoliths as in the case of the selective extraction of berberine in plasma and urine samples. ${ }^{26}$

In all these works where purification or selective extraction of the analyte is aimed, the tip contains a selective media for retention and further elution. Alternatively, the tip could retain impurities, dispensing a cleaner solution containing the species of interest. There is not any case reported in the bibliography in which a detectable molecule is included in the tip-based assay.

We propose here, for the first time, the use of micropipette tips for performing an immunoassay with analytical purposes. The assay is aimed to determine the concentration of the analyte and not just to purify it. Thus, after capturing the analyte, incubation with a detection antibody (labelled with an appropriate enzyme) occurs. Later, an enzymatic interaction takes place and a detectable electroactive species is produced. The final solution is deposited on a paper-based electrochemical platform, that includes staples as electrodes, and the detection is performed. In this way, in another out-of-box application, the micropipette tip is not only the element for dispensing solutions but also the scaffold where the immunoassay takes place. It is important to note that in most electrochemical immunosensors, recognition and transduction happen on the same surface. This fact may produce poisoning of electrodes and in many cases, both steps are separated ${ }^{27,28}$ to avoid protein adsorption and surface passivation.

Celiac disease (CD) is a frequent disorder affecting the small intestine in people with genetic predisposition, with a prevalence of $1 \%$ of the population. ${ }^{29}$ It is caused by an abnormal immune response to wheat, barley and rye proteins. It presents various symptoms such as malabsorption, diarrhea, weight loss, osteoporosis, fatigue and abdominal discomfort and nowadays, the only treatment is a life-long gluten free diet. ${ }^{30}$ Different tests are carried out to diagnose the disease, ranging from intestinal biopsies to serological tests. Celiac patients without treatment usually present an increase of immunoglobulins of classes A $(\operatorname{IgA})$ and $\mathrm{G}(\mathrm{IgG})$ that reacts against wheat gluten or against an autoantigen called transglutaminase (TG).

TG is an enzyme found both within and outside the cells, ${ }^{31}$ which is involved in various processes in the celiac disease. It produces the deamination of key glutamine residues present in gluten peptides, transforming the gluten protein in another much more reactive. On the other hand, TG is involved in a humoral autoimmune response resulting in antibodies secretion and circulation, mainly IgAs, turning that into a specific (95-99\%) serological celiac disease biomarker. ${ }^{32}$ These immunoglobulins can be easily quantified via ELISA (enzyme-linked imunoabsorbent assay).

As can be found in the bibliography, electrochemical immunosensors for celiac disease diagnosis have been employed using different surfaces. Conventional glassy carbon ${ }^{33}$ and screen-printed carbon electrodes ${ }^{34}$ have been nanostructured with gold nanoparticles for the multiplexed detection of $\operatorname{IgA}$ and IgG. Gold nanoelectrodes fabricated on a polycarbonate membrane allowed segregating biorecognition and electron transfer events in both, electrochemical ${ }^{35}$ and electrochemiluminescent ${ }^{36}$ approaches. Although alkaline phosphatase has been used as enzymatic label ${ }^{33}$, horseradish peroxidase has been the most common, using $\mathrm{H}_{2} \mathrm{O}_{2}$ as substrate and thionine ${ }^{34}$ or hydroquinone $\mathrm{S}^{35}$ as mediators. In this work, we have used micropipette tips as a new ELISA platform for the detection of anti-tTG $(\operatorname{IgA})$, with HRP and TMB- $\mathrm{H}_{2} \mathrm{O}_{2}$ as enzyme/substrate system for the detection on a paper-based platform. This leads to a low-cost (in comparison with other celiac disease electrochemical biosensors) and simple device with huge opportunities in the global market, since micropipette tips are common materials in any laboratory. Developing this assay in micropipette tips would become in a major breakthrough since the total cost will be reduced and the training is minimum, being possible to apply it in the developing areas where an efficient disease diagnosis (North Africa has a population with the highest prevalence of CD known in the world $\left.(5.6 \%)^{37}\right)$ is really needed. Not only it will be useful for the diagnosis of the celiac disease, but also for the most common infectious diseases with prevalence in the most unfavorable areas such as malaria, HIV or tuberculosis ${ }^{38}$. In addition, the integration with an electrochemical staple-based platform fits with the purposes of miniaturization of the whole analytical process.

\section{EXPERIMENTAL SECTION}

Poly-L-lysine hydrochloride (mol. wt. 30,000-70,000), bovine serum albumin (BSA), $\alpha$-Casein, 4-(2-hydroxyethyl)-1piperazineethanesulfonic acid (HEPES), and sodium tetraborate were provided by Sigma Aldrich. Sulfuric acid, sodium hydroxide, sodium chloride, sodium hydrogen carbonate, sodium carbonate and Tween 20 were provided by Merck Group. Sodium dihydrogen phosphate dihydrate and sodium hydrogen phosphate anhydrous were provided by VWR Chemicals. Phosphoric acid was provided by Panreac and recombinant human tissue transglutaminase (tTG) was purchased from MyBioSource. Human plasma was provided by Merck (P9523).

Anti-tissue transglutaminase (anti-tTG) IgA, anti-IgA labelled with horseradish peroxidase (HRP) and the substrate solution $\left(3,3^{\prime}, 5,5^{\prime}\right.$-tetramethylbenzidine, TMB, with $\left.\mathrm{H}_{2} \mathrm{O}_{2}\right)$ were part of two commercial kits employed for the detection of anti-tTG IgA (Kit Varelisa $^{\mathrm{TM}}$ Celikey $^{\circledR}$ from Phadia and TTG-IgA ELISA kit from MyBioSource). A substrate solution containing TMB was also provided by Sigma. A $1 \mathrm{mg} / \mathrm{mL}$ poly-L-lysine hydrochloride stock solution was prepared in $10 \mathrm{mM}$ PBS (137 $\mathrm{mM} \mathrm{NaCl}, 2.7 \mathrm{mM} \mathrm{KCl}) \mathrm{pH} 7.4$ and stored at $-20^{\circ} \mathrm{C}$. Working solutions $(10 \mu \mathrm{g} / \mathrm{mL})$ were obtained by diluting the stock solution in $10 \mathrm{mM}$ PBS $\mathrm{pH}$ 7.4. A volume of $100 \mu \mathrm{L}$ of original 
recombinant human tissue transglutaminase (tTG) was reconstituted in Milli-Q water to obtain $1 \mathrm{mg} / \mathrm{mL}$ solution. After aliquoting it, a $5 \mu \mathrm{g} / \mathrm{mL}$ tTG working solution was prepared in $100 \mathrm{mM}$ carbonate buffer $\mathrm{pH}$ 9.6.

Different blocking buffer-protein mixtures were evaluated: 10 $\mathrm{mM}$ PBS pH 7.4 with $5 \%$ of bovine serum albumin (BSA), 10 $\mathrm{mM}$ borate buffer $\mathrm{pH} 8.4$ with $2 \%$ of $\alpha$-casein, $10 \mathrm{mM}$ borate buffer $\mathrm{pH} 8.4$ with 5\% BSA, 10 mM HEPES buffer $\mathrm{pH} 7.4$ with $2 \%$ of $\alpha$-casein and $10 \mathrm{mM}$ HEPES buffer $\mathrm{pH} 7.4$ with $5 \%$ BSA. The blocking buffer was prepared daily. On the other hand, a 10 $\mathrm{mM}$ PBS pH 7.4 with $5 \%$ of Tween 20 was used as washing buffer. A $2 \mathrm{M} \mathrm{H}_{2} \mathrm{SO}_{4}$ was used as stop solution.

Throughout this work 1-200 $\mu \mathrm{L}$ polypropylene tips (TIPD-200$1 \mathrm{~K} 0$ Premium Line, Labbox) were used. Also, $26 \mathrm{~mm} \times 0.59$ $\mathrm{mm}$ stainless-steel pins (Folch Metalurgia) were used to seal the bottom of the tips during the immunoassay. For the amperometric measurement, a $\mu$ AUTOLAB TIPE III potentiostat was used. It was handled by NOVA 2.1 software (Metrohm Autolab). Millipore Direct-Q(R) $3 \mathrm{UV}$ was used to obtain purified water.

Immunoelectrochemical assay

Immobilization of the capture bioreagent. Micropipette tips were first modified aspirating $20 \mu \mathrm{L}$ of a $10 \mu \mathrm{g} / \mathrm{mL}$ poly-L-lysine solution. They were sealed at the bottom with a stainless-steel pin and at the top with Parafilm ${ }^{\circledR}$ and were left at $37^{\circ} \mathrm{C}$ in the stove overnight.

After that, the poly-L-lysine solution was thrown away and the tips were washed three times with the washing buffer. Then, they were filled again with $20 \mu \mathrm{L}$ of the $5 \mu \mathrm{g} / \mathrm{mL}$ tissue transglutaminase solution in $100 \mathrm{mM}$ carbonate buffer $\mathrm{pH}$ 9.6. They were sealed again and incubated overnight at $4^{\circ} \mathrm{C}$. After three washings, $20 \mu \mathrm{L}$ of the blocking buffer were aspirated and tips were incubated overnight at $4^{\circ} \mathrm{C}$ and finally washed. At this step tips are ready (modified with the capture reagent and blocked) for immunodetection of anti-tTG (IgA).

All these steps were optimized to find the best concentrations and reaction times.

Immunoassay. For the ELISA, two different commercial kits for anti-tTG immunoglobulin A determination were used. Although first trials were done with a kit Varelisa ${ }^{\mathrm{TM}}$ Celikey $^{\circledR}$ from Phadia, the final results were achieved using a TTG-IgA ELISA kit from MyBioSource. Commercial kit provided us with four different anti-tTG (IgA) standards, with concentrations ranging between 12.5 and $100 \mathrm{U} / \mathrm{mL}$. A negative control $(0$ $\mathrm{U} / \mathrm{mL}$ standard) was also provided. For the immunoassay, a volume of $20 \mu \mathrm{L}$ of a 1:2 cocktail of anti-tTG IgA standard and anti-IgA labelled with HRP was aspirated. This solution was incubated in modified micropipette tips for $3 \mathrm{~h}$ at $37^{\circ} \mathrm{C}$. After this time, tips were washed with the washing buffer and later, 20 $\mu \mathrm{L}$ of the kit substrate solution $\left(\mathrm{TMB} / \mathrm{H}_{2} \mathrm{O}_{2}\right)$ were aspirated and left in the dark for $5 \mathrm{~min}$ at room temperature. This time was enough to convert enzymatically TMB into a blue colored product. A scheme of the immunoassay can be observed in Figure 1A.

Results obtained for positive and negative controls were compared with those obtained following the ELISA procedure specified in the TTG-IgA ELISA kit (MyBioSource). Optical readout was made with a microplate spectrophotometer (Eon $\mathrm{TM}$, BioTek) at $450 \mathrm{~nm}$.

Electrochemical measurements. The blue solution obtained after performing the immunoassay in the micropipette tip was transferred to a microcentrifuge tube containing $1 \mu \mathrm{L}$ of $2 \mathrm{M}$ $\mathrm{H}_{2} \mathrm{SO}_{4}$. This stopped the enzymatic oxidation of TMB and stabilized it for electrochemical measurements. These were done on a low-cost device based on stainless-steel staples previously developed in our research group. ${ }^{17}$ This device uses disposable staples as electrodes (the one acting as working electrode modified with carbon ink) combined with paper strips where the sample is deposited. A volume of $18 \mu \mathrm{L}$ of the solution was added to the low-cost staple-based platform and a chronoamperogram was recorded at $-0.1 \mathrm{~V}$ for $50 \mathrm{~s}$. Then, the current due to the reduction of the previously enzymatically oxidized TMB was measured.

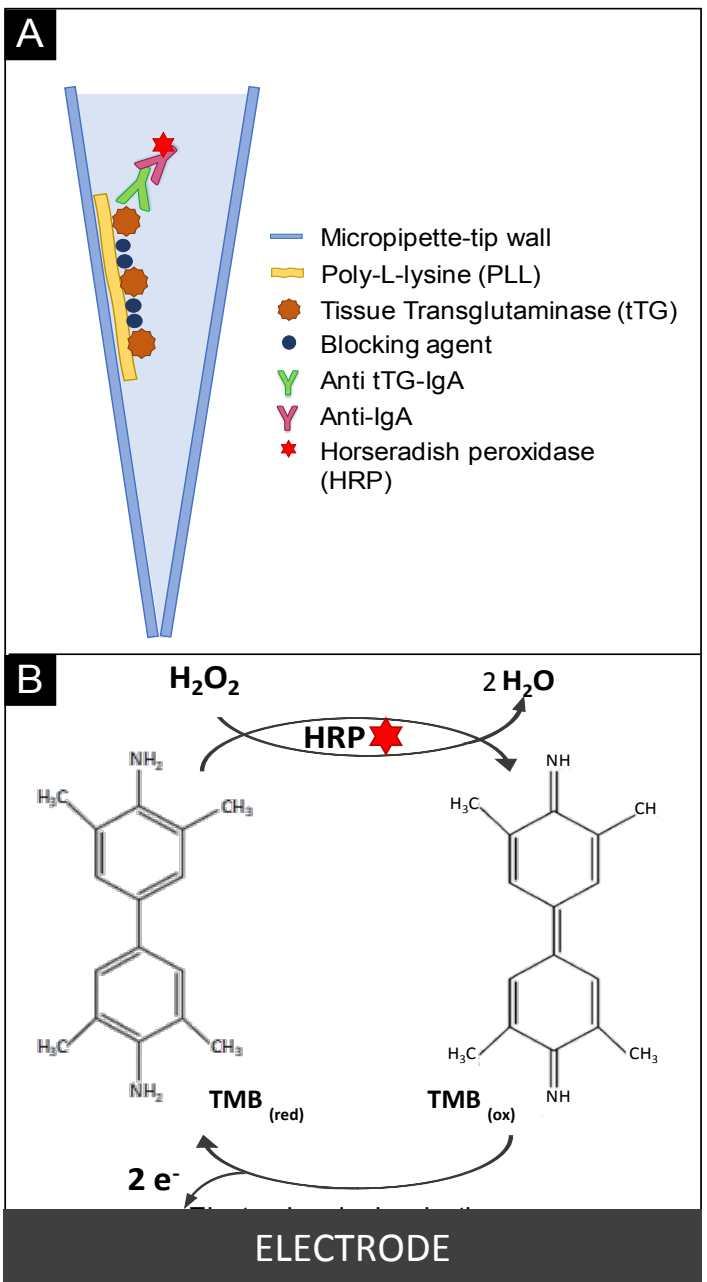

Figure 1. A) Schematics of the immunoassay performed in the micropipette tip. B) Scheme of the enzymatic and electrochemical processes involved.

\section{RESULTS AND DISCUSSION}

Micropipette tips were employed for the first time with the purpose of performing immunoassays, which detection was accomplished by electrochemical measurements. In this case, the antigen tTG (capture bioreagent) was immobilized on the wall of 
the tips, and an immunometric methodology was followed for the determination of anti-tTG antibodies. The immobilisation of the capture reagent (tTG) was a very relevant step for obtaining precise and accurate results. Then, in the first place, the ability of the micropipette tips to fix biological material in their internal wall was studied. In this case, 1-200 $\mu \mathrm{L}$ polypropylene (PP) micropipette tips were used. Although many different materials for this type of consumable already exist, polypropylene has clear advantages in comparison with others such as polystyrene (PS), polyethylene (PE) or polycarbonate (PC). PP is more stable and transparent (quite useful for visual evaluation) than PE. It has better chemical resistance, lower biomolecule binding than PS and PC and it can be used across a broader temperature range than PS.

In terms of volume, the $1-200 \mu \mathrm{L}$ tips were filled with the solution $10 \%$ of their volume $(20 \mu \mathrm{L})$ in every step. It should also be taken into consideration the conical shape of the micropipette tip, smaller at its base (i.d of $0.59 \mathrm{~mm}$ ) and wider at the top (i.d of $5 \mathrm{~mm}$ ). When $20 \mu \mathrm{L}$ of the solution were aspirated, $1.9 \mathrm{~cm}$ of the tip length was full of the solution.

As mentioned before, one of the common requirements for the tips is low adsorption of the analyte, so, the ability of these consumables to fix molecules on their inner wall is almost negligible. Then, to immobilize the capture reagent (a protein), modification with poly-L-lysine (PLL), a lysine homopolymer commonly used in cell cultures and ELISA plates to improve the adherence of the cells or capture reagents to the surface ${ }^{39}$, was evaluated. Electrostatic interactions between the polymer and proteins will mainly depend on the $\mathrm{pH}$ and the isoelectric point of both. Thus, when a layer of cationic PLL is present, it will contribute to create positive charges on the surface making possible the attraction to any negative charge ${ }^{40}$. But, apart from ionic interactions that will essentially depend on $\mathrm{pH}$, tissue transglutaminase (tTG) has probed to have the function to crosslink primary amines (such as lysine) and peptide-bound glutamine ${ }^{41}$. Transglutaminases are acyl transferases that catalyze formation of amide bonds between the $\gamma$-carboxyl group of glutaminyl residues and a primary amino group, usually the $\varepsilon$ amino group of a lysyl residue ${ }^{42}$. Indeed, such cross-linking reaction catalyzed by $\mathrm{TG}$ has been recently exploited for immobilization and electrochemical sensing of gliadin ${ }^{43}$. Transglutaminase reacts with gliadin and also promotes crosslinking between gliadin and collagen ${ }^{44}$. However, this binding does not occur in the absence of transglutaminase ${ }^{45}$. On the other hand, tTG has been successfully incorporated in a network composed by casein and PLL, these polymers being cross-linked by the action of tTG itself ${ }^{46}$. We hypothesize that, in our case, at first PLL interacts with tTG by non-covalent interactions (H-bonding, local ionic and hydrophobic interactions, van der Waals forces etc.) and later on, in an overnight blocking step with casein (see below), this protein is cross-linked to PLL by tTG action, to form a network that strengthen the immobilization of tTG.

To prepare the poly-lysine layer, $20 \mu \mathrm{L}$ of a $10 \mu \mathrm{g} / \mathrm{mL}$ PLL solution (in $10 \mathrm{mM}$ PBS $\mathrm{pH}$ 7.4) was aspirated and, after introducing a pin $(\varnothing 0.59 \mathrm{~mm})$ at the bottom end to avoid leaking, the tip was separated from the micropipette and left to incubate overnight at $37^{\circ} \mathrm{C}$. It is important to note that, since this is a long step and in order to avoid the evaporation of the solution from the tip, the top was sealed with Parafilm ${ }^{\circledR}$. With both ends closed, the tip can be handled easily without the micropipette. To confirm that the polymeric modification succeeded, $20 \mu \mathrm{L}$ of the standard solution of anti-IgA labeled with HRP (reagent provided with the commercial kit) were aspirated and left to incubate overnight at $37^{\circ} \mathrm{C}$. After appropriate washing, $20 \mu \mathrm{L}$ of the substrate solution (as provided) containing TMB (3,3',5,5'-tetramethylbenzidine) and $\mathrm{H}_{2} \mathrm{O}_{2}$ was left to interact for $5 \mathrm{~min}$. If PLL is present and proteins (anti-IgA labeled with HRP) have been immobilized, different measurements should be obtained for coated and uncoated tips.

Horseradish peroxidase (HRP) catalyzes the redox reaction $\mathrm{H}_{2} \mathrm{O}_{2}$ $+\mathrm{SH}_{2}$---> $2 \mathrm{H}_{2} \mathrm{O}+\mathrm{S}$, schematized in Figure $1 \mathrm{~B}$ for $\mathrm{SH}_{2}$ being TMB. The use of this molecule as electrochemical substrate for HRP was investigated some decades ago with a competitive ELISA for rabbit IgG. ${ }^{47}$ When $\mathrm{TMB}_{\text {(red) }}$ is incubated in the presence of this enzyme and $\mathrm{H}_{2} \mathrm{O}_{2}$, it is oxidized into a blue product $\left(\mathrm{TMB}_{(\mathrm{ox})}\right)$, that turns into yellow after stopping the reaction with concentrated $\mathrm{H}_{2} \mathrm{SO}_{4}$ (protonated form of the product). This is the one used to be optically detected in common ELISA immunoassays. Aromatic amines of the benzidine series (as $\mathrm{TMB}_{\text {(red) }}$ in Figure 1B) form typical reversible oxidation-reduction systems with their oxidation products, the benzidinequinonediimines $\left(\mathrm{TMB}_{(\mathrm{ox})}\right.$ in Figure 1B $)^{48}$. Two different modes of oxidation of the amines occur in the strongly acid and slightly acid solutions. In the former, oxidation is a simple bivalent process, whereas in the latter it involves intermediate free radicals and follows a univalent course. When radicals of half-oxidized semiquinones can be stabilized by resonance, oxidation can take place via radical intermediates. These $p$-quinonediimines are easily hydrolyzed to quinones, hydrolysis being accelerated by increase in the $\mathrm{pH}$. However, the oxidation products of the amines in strongly acid solutions are extremely stable. This may be accounted for by the assumption that polymerization and substitution reactions, which the quinonediimines undergo at higher $\mathrm{pH}$ values, are made impossible by the completely ionic characters of the imine in solution $\left(=\mathrm{NH}_{2}{ }^{+}\right)$. On the other hand, substituents groups, particularly when attached directly to the quinonoid nucleus (as the methyl groups in this case) have a pronounced effect on the potential. Indeed, para-directing groups, such as $-\mathrm{CH}_{3}$, lower the potential. ${ }^{48}$

In Figure 2 the difference between a non-covered tip (Figure 2A.1) and a previously PLL-covered tip (Figure 2A.2) can be observed. In the last one, an intense blue color can be seen inside the tip after $5 \mathrm{~min}$. This is due to the oxidation of TMB by the bioconjugated HRP. However, in the non-covered tip (Figure 2A.1) no color was obtained, which means that no HRP was fixed to the internal wall. From this, it can be deduced that: i) it is necessary to perform a previous tip coating in order to fix the capture molecules to the inner wall, ii) PLL is a good modifier that favors the immobilisation of molecules and iii) non-specific adsorption is not significant when PLL is not present.

Although optical detection is not aimed, the intensity of the color of the enzymatic product can be employed to follow the immunoassay. A darker blue color is indicative of a higher amount of oxidized TMB. This means the presence of a higher amount of HRP and thus, of detection antibody. Since this is a sandwich format, this implies a higher concentration of anti-tTG present in the sample. However, quantification is made 
electrochemically. The color of the tip (although colorless tips are also available), as well as its conical shape, does not make this approach useful for "on-the-tip" optical detection. However, none of these issues interfere with the electrochemical detection, which in the amperometric mode only requires an electroactive species (oxidized form of TMB) and a conductive surface for the electron transfer. Although further integration of the electrochemical detection is aimed, the first step is the optimization of the immunoassay and therefore, an "off-the-tip" detection is here presented. If optical readout were to be employed, the use of the tip would not introduce any additional benefit over well-established microtiter plates, which apart from detection cuvettes are also the solid support for the immunoassay. In the case of the electrochemical detection, a small volume is deposited on the paper-based and low-cost platform for direct measurement with simple instrumentation, which can be easily adapted for decentralized analysis. On the other hand, it has been reported that better performance was achieved with amperometric than spectrophotometric (fluorimetric) detection using TMB in a competitive ELISA for rabbit IgG. ${ }^{47}$

Color development can occur also due to nonspecific binding (NSB) between the analyte (anti-tTG) or subsequent immunoreagents and the solid phase to which the receptor (tTG) is immobilized. The next important step was to carry out an adequate blocking step to inhibit this interaction, so that only specific occurred. Although this step has to be performed after the immobilization of $\mathrm{tTG}$, we have evaluated the maximum non-specific signal that the labelled secondary antibody could produce. Proteins (e.g., BSA and casein) are believed to fill in stretches of the solid phase not occupied by the immobilized receptor ${ }^{49}$. The effectivity will depend on the immunoassay, the blocking agent but also on the solid phase. In this case, the tip, a non-porous polymeric substrate covered with PLL, was treated with different buffer solutions containing BSA or casein. Thus, a $5 \%$ of BSA has been added to: i) $10 \mathrm{mM}$ PBS pH 7.4, ii) $10 \mathrm{mM}$ HEPES buffer $\mathrm{pH} 7.4$ and iii) $10 \mathrm{mM}$ borate buffer $\mathrm{pH} 8.4$. Similarly, a $2 \%$ of casein has been added to: iv) $10 \mathrm{mM}$ HEPES buffer $\mathrm{pH} 7.4$ and v) $10 \mathrm{mM}$ borate buffer $\mathrm{pH} 8.4$. Concentrations for proteins blocking agents were chosen according to different immunoassay protocols. In order to evaluate NSBs, $20 \mu \mathrm{L}$ of each blocking solution were aspirated and left to incubate overnight at $4^{\circ} \mathrm{C}$. After another washing, the HRP-conjugated anti-IgA was incubated and the TMB reaction was performed as explained previously. It is important to note that washing is an important step at heterogeneous immunoassays and, with the method developed in this work, aspiration and dispensation are simple steps that can be easily performed with micropipette tips.

A buffer that blocks correctly the surface avoids the non-specific binding of antibodies and thus no color development should be seen after TMB incubation. From the five blocking buffers tested, $10 \mathrm{mM}$ PBS pH 7.4 with $5 \%$ of BSA and $10 \mathrm{mM}$ borate buffer $\mathrm{pH} 8.4$ with $2 \%$ of casein gave the best results. Both were checked again but performing the blocking after tTG immobilization.

Commercial kits for the detection of anti-tTG (IgA) were used for this work. They were thought to be used in combination with microtiter ELISA plates that had the antigen (tTG) immobilized. For the tip (PLL-covered), we aspirated $20 \mu \mathrm{L}$ of a $5 \mu \mathrm{g} / \mathrm{mL}$ tTG solution in $100 \mathrm{mM}$ carbonate buffer $\mathrm{pH} 9.6$ and left to interact overnight at $4^{\circ} \mathrm{C}$. Results obtained after washing, blocking, incubating with HRP-conjugated anti-IgA and adding TMB are shown in Figure 2B. Here, pictures of the tips with and without these two blocking agents can be seen. It can be observed that, with the blocking buffer based on casein (Figure 2B.1) there is no blue color development meanwhile the blocking buffer based on BSA (Figure 2B.2) produces a lighter blue color. As commented before, transglutaminases are acyl-transferases, that can cross-link proteins (through their glutamine residues) to primary amines (as e.g. poly-L-lysyl residues) ${ }^{42}$. They are calcium-dependent enzymes, but in some cases they can act without added metal ion $^{50}$. Moreover, they display differences in specificity regarding the glutamine residues available. ${ }^{42}$ This could be the reason of the difference observed in the blocking with BSA or casein. Indeed, it has been reported that when a mixture of $\alpha$-casein and $\beta$-lactoglobulin was used as substrate, preferential cross-linking of $\alpha$-casein occurred with an immobilized enzyme. ${ }^{46}$ In any case, the distinction with the unblocked tip (Figure 2B.3) is clear. According to this, the blocking buffer based on casein was chosen for the remaining of the work.

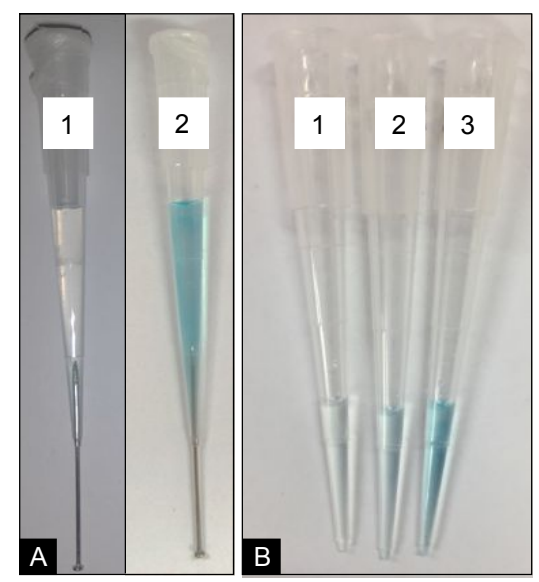

Figure 2. Pictures of micropipette tips incubated with HRPlabelled anti-IgA after TMB reaction: A) to compare PLL modification: 1) non-PLL-covered tip and 2) previously PLLcovered tip. B) to compare PLL-covered tips with the capture reagent immobilized when: 1) they have been blocked overnight with $10 \mathrm{mM}$ borate buffer $\mathrm{pH} 8.4$ with $2 \%$ of casein, 2) they have been blocked overnight with $10 \mathrm{mM}$ PBS pH 7.4 with 5\% of BSA and 3) a blocking step is not performed.

At this point, with PLL-modification, tTG-immobilization and blocking steps performed, the tip was prepared for the immunoassay. The next steps are the incubation with the sample/standard solution containing the analyte and the addition of the detection antibody. These steps can be done sequentially or both reagents can be added together. To simplify the procedure, a solution containing anti-tTG (IgA) and HRPlabelled anti-IgA was prepared, aspirated and incubated in the tip. This is an important step since the kinetics of antigenantibody reactions is such that, in most cases, cross reactivity decreases with incubation time and is minimal when equilibrium is reached. ${ }^{51}$ With the aim of studying the effect of the 
incubation time, a volume of $20 \mu \mathrm{L}$ of a 1:2 cocktail of anti-tTG (IgA) standard $(25 \mathrm{U} / \mathrm{mL})$ and anti-IgA labelled with HRP were absorbed and left to incubate at $37^{\circ} \mathrm{C}$ during $30 \mathrm{~min}, 1,2$ and $3 \mathrm{~h}$ in different tips. After TMB reaction, only for the $3 \mathrm{~h}$ incubation tip the blue color was achieved. Thus, an incubation time of $3 \mathrm{~h}$ for fixing the antibody was chosen for the rest of the experiments.

Even when tip preparation (modification, immobilization and blocking) may seem time consuming, they could be massproduced and left ready for the immunoassay when needed. Preliminary studies on the stability of the functionalized tips has been made and tips with PLL-modification, tTG-immobilization and blocking have been stored completely sealed and with desiccant bags at $4^{\circ} \mathrm{C}$ for one week. The tips maintained a $70 \%$ of the initial signal with a RSD of $6 \%$ (data not shown). Thorough studies should be made in case commercialization is aimed.

All the steps of the immunoassay, including time and temperature, are schematized in Table 1. It is important to note that after each step, a washing with $10 \mathrm{mM}$ PBS pH 7.4 with 5\% of Tween 20 was done in triplicate.

Table 1. Scheme of the entire immunoassay steps with the incubation temperature and time of each step.

\begin{tabular}{ccc}
\hline Step & $\begin{array}{c}\text { Incubation } \\
\text { temperature }\end{array}$ & $\begin{array}{c}\text { Incubation } \\
\text { time }\end{array}$ \\
\hline PLL coating & $37^{\circ} \mathrm{C}$ & Overnight \\
tTG immobilisation & $4^{\circ} \mathrm{C}$ & Overnight \\
Blocking with casein & $4^{\circ} \mathrm{C}$ & Overnight \\
Anti-tTG and anti-IgA- & $37^{\circ} \mathrm{C}$ & $3 \mathrm{~h}$ \\
HRP incubation & & $5 \mathrm{~min}$ \\
Enzymatic reaction & Room temperature & \\
(TMB substrate) & & \\
\hline
\end{tabular}

At this point, it is time to select a detection system that allows quantitative measurements. Electrochemical detection is very appropriate, having advantages such as the use of miniaturized equipment and simplicity while maintaining sensitivity and precision. As indicated above, we have employed a staple-based device previously developed in our research group. It consists of three stainless-steel staples used as electrodes and wax-sealed paper strips acting as the porous platform where the solution is deposited. ${ }^{17}$ The electroactive species is the product of the enzymatic oxidation of TMB (HRP catalyzes the oxidation of benzidines in the presence of $\left.\mathrm{H}_{2} \mathrm{O}_{2}\right)^{47}$ and the analytical signal is the current due to its electrochemical reduction (as shown in Figure 1B). Consequently, the higher the reduction signal, the higher the concentration of previously oxidized TMB and, at the end, more concentration of anti-tTG $(\operatorname{IgA})$ in the original sample. The time of the enzymatic reaction should be controlled to warranty precision of the analytical signals.

To ensure that all the measurements were taken at the same reaction time: i) the solution with the product of the enzymatic reaction can be deposited directly on the paper-based platform for measurement or, ii) the reaction can be stopped by adding an acidic solution. Since the inner solution is going to be deposited in the electrochemical platform, the stopping solution is not required. However, measurements were performed following both approaches (depositing the solution directly on the paperbased platform or stopping the reaction with $1 \mu \mathrm{L}$ of $2 \mathrm{M}$ $\mathrm{H}_{2} \mathrm{SO}_{4}$ ) and those recorded when sulphuric acid was added were found to be more precise. Moreover, the electroactivity of TMB changed considerably in acidic $\mathrm{pH}$ values, as can be seen in Figure $\mathrm{S} 1$. A cyclic voltammogram recorded in a TMB substrate solution (Figure S1A), provided with the kit, and a cyclic voltammogram of the same solution in $2 \mathrm{M} \mathrm{H}_{2} \mathrm{SO}_{4}$ (Figure S1B) are shown. It can be observed that in the first case two electrochemical processes are present (two subsequent oneelectron processes ${ }^{47}$ ) and however, only one reversible process (one two-electron process ${ }^{48}$ ) occurs when a strongly acidic medium was added. These processes, due to TMB $\left(\mathrm{H}_{2} \mathrm{O}_{2}\right.$ would need higher overvoltages ${ }^{52}$ ) are in accordance with that explained before for the redox process of TMB, that could happen in one or two-steps depending on the $\mathrm{pH}$ of the medium. In this case, the process of analytical interest (reduction of the enzymatically produced $\mathrm{TMB}_{(\mathrm{ox})}$ ) is electrochemical. Although techniques such as cyclic voltammetry and also square wave voltammetry (Figure S1C) could be employed as much more selective than chronoamperometry, we have solved the selectivity issue by employing a biorecognition system. The sample is aspirated into the tip and the anti-tTG is selectively captured by the immobilized tTG. Other molecules that could interfere electrochemically would not interact and then, chronoamperometry (CA), a very simple technique, appropriate for miniaturized devices can be employed. Taking into account Figure S1B, if a potential of $-0.1 \mathrm{~V}$ was applied to the working electrode $v s$. a stainless-steel staple pseudoreference electrode, a cathodic current due to the reduction of the enzymatically oxidized TMB was produced (no electrochemical oxidation occurred at this potential). This is a potential negative enough to produce electron transfer in a process that will be limited by mass transport. Moreover, being the potential a little more negative than required, possible small variations due to the use of a pseudoreference electrode will not prevent the reduction to take place. The cathodic current (measured at 50s) was the analytical signal correlated with analyte concentration.

To record the chronoamperograms after performing the immunoassay, the volume of $20 \mu \mathrm{L}$ contained in the tip was poured in a microcentrifuge tube where $1 \mu \mathrm{L}$ of the stopping solution was already located. Finally, after homogenization, 18 $\mu \mathrm{L}$ of this final solution were deposited on the corresponding paper strip area of the electrochemical platform and the reduction at $-0.1 \mathrm{~V}$ was recorded for $50 \mathrm{~s}$. In order to know if the analytical signal was correlated with the concentration of antitTG (IgA), a calibration curve was performed between 0 and 100 $\mathrm{U} / \mathrm{mL}$, using the standard solutions provided in the commercial kit. The current measured at $50 \mathrm{~s}$ correlated linearly with the concentration of the analyte, following the equation $\mathrm{I}_{50 \mathrm{~s}}-\mathrm{I}_{0}(\mu \mathrm{A})$ $=-0.0033 \mathrm{C}(\mathrm{U} / \mathrm{mL})-0.0117$, with a $\mathrm{R}^{2}$ of 0.995 , where $\mathrm{I}_{0}$ is the intensity of the current for the blank (also for $50 \mathrm{~s}$ ). A limit of detection (LOD) of $4.6 \mathrm{U} / \mathrm{mL}$, calculated as the concentration corresponding to 3 times the standard deviation of the intercept was obtained. The provider of the spectrophotometric kit commercialized for this IgA detection establishes values below 5 $\mathrm{U} / \mathrm{mL}$ as negative, and then all the samples with values 
(electrochemical detection) below the LOD would be considered negatives. In the Figure 3A, the tips containing the product of the immunoassay before stopping the enzymatic reaction with 2 $\mathrm{M} \mathrm{H}_{2} \mathrm{SO}_{4}$ are shown. It can be observed that in the first micropipette tip no color is obtained and, as the anti-tTG (IgA) concentration increases, the blue color becomes more intense. In Figure $3 \mathrm{~B}$, the calibration graph obtained from chronoamperograms recorded for the different concentrations of anti-tTG $(\operatorname{IgA})$ is shown.

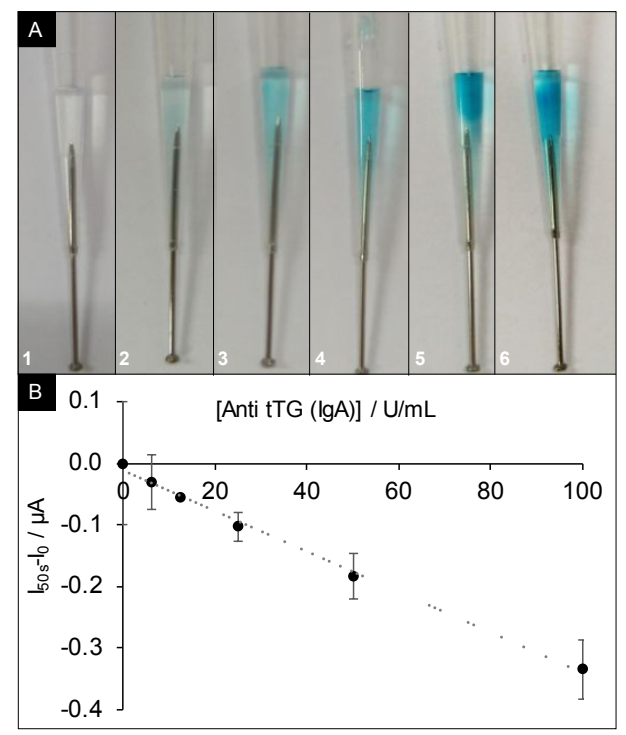

Figure 3. A) Pictures of the micropipette tips after performing immunoassays with increasing anti-tTG (IgA) concentration: 1) $0,2) 6.25,3) 12.5$, 4) 25,5$) 50$ and 6) $100 \mathrm{U} / \mathrm{mL}$. B) Calibration graph of the micropipette tip immunoassay. Each measurement was done in triplicate (using different tips for each measurement, as they are not reusable). Error bars correspond to the standard deviation of the measurements .

In order to verify the reliability of the methodology, it was compared with a commercial ELISA performed in microplate (TTG-IgA ELISA kit, MyBioSource) and detected optically. Figure S2 shows the calibration curve obtained with the commercial ELISA. With the aim of validating the methodology, the sample diluent provided with the kit was spiked with anti-tTG IgA standard to obtain different concentrations. Thus, one negative and two positive controls were evaluated. In Table 2 , the results obtained with the immunoassay performed in the micropipette tip (electrochemical readout) as well as those obtained with the commercial ELISA (optical readout) are reported.

Table 2. Results obtained for negative and positive controls with the immunotip and electrochemical readout and the commercial ELISA with optical detection.

\begin{tabular}{c|ccc} 
& [Anti-tTG] & Immunotip & ELISA \\
& $U / m L$ & $U / m L$ & $U / m L$ \\
\hline Control 1 (-) & 0 & Below LOD & Below LOD
\end{tabular}

\begin{tabular}{l|lll} 
Control 2 (+) & 33 & $33 \pm 4.2$ & $33 \pm 1.3$ \\
Control 3 (+) & 71 & $66 \pm 2.3$ & $71 \pm 5.0$
\end{tabular}

The provider establishes values below 5 and over $8 \mathrm{U} / \mathrm{mL}$ for negative and positive controls, respectively. In the case of the positive controls, a $t$-student test was performed to evaluate the absence of systematic errors. Considering a level of confidence of $98 \%$ and 3 degrees of freedom, we can conclude that there are no significant differences between both methodologies. A negative value for the concentration of the negative control is obtained with the immunotip. Since the intercept of the calibration curve is negative, an intensity of the current above this value will generate negative concentrations, which in any case are below the LOD. To get closer to real sample analysis, five positive controls were prepared by spiking artificial human plasma with anti-tTG IgA standards. Results obtained following the electroanalytical methodology were compared with those derived from the optical readout and commercial ELISA microplates. The results for the immunoassays performed in the micropipette tips were $17 \pm 3.2 \mathrm{U} / \mathrm{mL}$ and $21 \pm 2.4 \mathrm{U} / \mathrm{mL}$ obtained with the optical readout.

Finally, the reproducibility of the assay was studied by measuring the reduction of TMB that has been enzymatically oxidized, in different tips. After performing the immunoassay in five micropipette tips for a $7 \mathrm{U} / \mathrm{mL}$ anti-tTG $(\operatorname{IgA})$ standard solution, a blue color was developed as shown in Figure 4A. In Figure $4 \mathrm{~B}$, pictures of five micropipette tip immunoassays for the blank $(0 \mathrm{U} / \mathrm{mL})$ are also shown. In both cases, chronoamperograms were recorded at $-0.1 \mathrm{~V}$ for $30 \mathrm{~s}$. The relative standard deviation (RSD) for the five measurements of anti-tTG was $8.9 \%$, which indicates the adequate precision of the methodology. The RSD for the blank measurements is $4.3 \%$. These results suggest that our tip-based assay could be used to easily and precisely determine anti-tTG $(\operatorname{IgA})$.

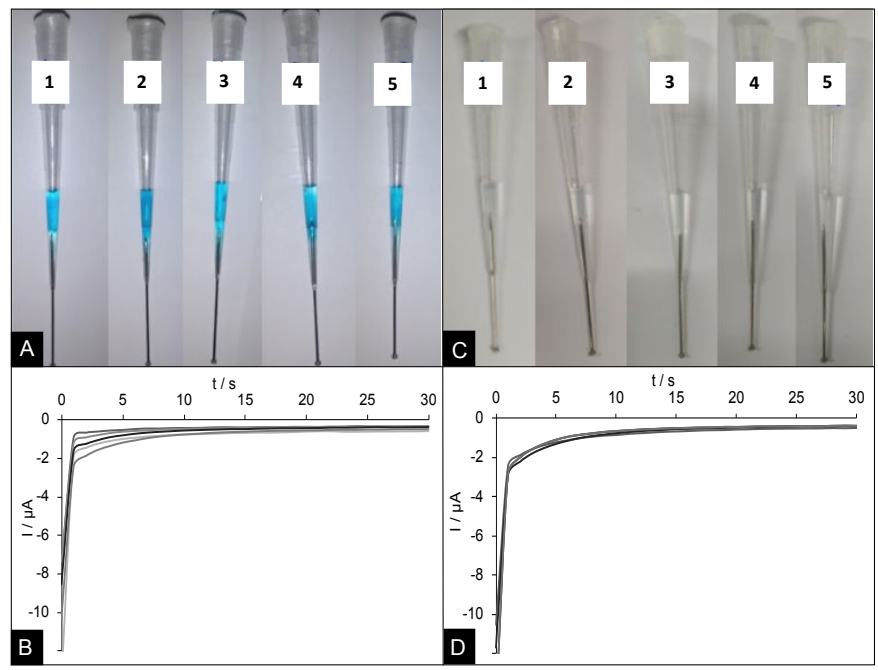

Figure 4. Study of the immunoassay reproducibility in five micropipette tips after $5 \mathrm{~min}$ of $\mathrm{TMB}$ reaction: A) with the presence $7 \mathrm{U} / \mathrm{mL}$ of anti-tTG (IgA) (from kit Varelisa ${ }^{\mathrm{TM}}$ Celikey) and C) without the analyte (blanks). The corresponding chronoamperograms $(-0.1 \mathrm{~V})$ are represented in B) and D). 


\section{CONCLUSIONS}

We have developed a new portable, low-cost immunoassay with a simple and easy-to-use electrochemical detection. This is the first time a micropipette tip is employed as a substrate for immunoanalytical purposes combined with a low-cost electrochemical detector. The study describes the development of an immunoassay using only polypropylene micropipette tips as immunoplatform and a simple paper-based device for detection.

This new methodology also presents advantages over the conventional immunoassays, since the tip/paper platform: i) uses low volume of reagents, being able to perform the assay with less than $25 \mu \mathrm{L}$, compared to the $100-150 \mu \mathrm{L}$ commonly required in conventional ELISA plates, ii) uses materials that are easy to transport and to store (both, tips and paper), iii) can be easily disposed after use, iv) can be mass-produced and v) is cost-effective $(\sim 0.008 €$ per device, excluding the biochemical reagents).

The combination with the electrochemical device maintains these advantages since a potentiostat is easy to transport and store, being yet simple and low-cost. On the other hand, one of the simplest electroanalytical techniques, chronoamperometry, is here employed for the determination of anti-tTG (IgA).

All the materials used for the development of this device (immuno and electroanalytical platforms) are commonly found in every laboratory, being possible to mass-produce and massmodify them. The linear relationship obtained between the intensity of the current and the anti-tTG concentration as well as the good precision of the methodology makes it very adequate for analytical purposes. Similar limits of detection were obtained with this methodology and the commercial ELISA with optical readout. Results for negative and positive controls evaluated were in accordance for both methodologies.

This design could be used for direct or indirect ELISAs with different electrochemical labels, as well as for the determination of different analytes of interest. Furthermore, it not only can provide a qualitative result (observing the colour by the naked eye), but also a quantitative one through the electrochemical detection. In this context, it is expected that this design would pave the way to the development of more micropipette tip-based immunoassays, adequate for the in-situ diagnosis of different diseases, being this very important not only for developing countries, but also in the biomedical area of the developed ones.

\section{ASSOCIATED CONTENT}

Supporting Information.

Cyclic voltammograms of $\mathrm{TMB} / \mathrm{H}_{2} \mathrm{O}_{2}$ recorded in $10 \mathrm{mM}$ PBS $\mathrm{pH} 7.4$ and $2 \mathrm{M} \mathrm{H}_{2} \mathrm{SO}_{4}$. Square wave voltammogram of $\mathrm{TMB} / \mathrm{H}_{2} \mathrm{O}_{2}$ recorded in $2 \mathrm{M} \mathrm{H}_{2} \mathrm{SO}_{4}$.

\section{AUTHOR INFORMATION}

Corresponding Author

*e-mail: mtfernandeza@uniovi.es

Author Contributions
All authors have given approval to the final version of the manuscript.

\section{Funding Sources}

This work has been supported by the FC15-GRUPIN-021 project from the Asturias Regional Government and the CTQ2014-58826-R project from the Spanish Ministry of Economy and Competitiveness (MEC). Andrea GonzálezLópez thanks the Consejería del Principado de Asturias for the award of her Severo Ochoa grant (BP17-36).

\section{ABBREVIATIONS}

PLL, poly-L-lysine; tTG, tissue transglutaminase; IgA, immunoglobulin A; ELISA, enzyme linked-immunoabsorbent assay; BSA, bovine serum albumin; TMB, 3,3',5,5'tetramethylbenzidine; HRP, horseradish peroxidase; CA, chronoamperometry.

\section{REFERENCES}

(1) Liu, B.; Du, D.; Hua, X.; Yu, X. Y.; Lin, Y. PaperBased Electrochemical Biosensors: From Test Strips to Paper-Based Microfluidics. Electroanalysis. 2014, 26 (6), 1214-1223.

(2) Ainla, A.; Mousavi, M. P. S.; Tsaloglou, M. N.; Redston, J.; Bell, J. G.; Fernández-Abedul, M. T.; Whitesides, G. M. Open-Source Potentiostat for Wireless Electrochemical Detection with Smartphones. Anal. Chem. 2018, 90 (10), 6240-6246.

(3) Martinez, A. W.; Phillips, S. T.; Whitesides, G. M.; Carrilho, E. Diagnostics for the Developing World: Microfluidic Paper-Based Analytical Devices. Anal. Chem. 2010, 82 (1), 3-10.

(4) Nie, Z.; Nijhuis, C. A.; Gong, J.; Chen, X.; Kumachev, A.; Martinez, A. W.; Narovlyansky, M.; Whitesides, G. M. Electrochemical sensing in paper-based microfluidic devices. Lab. Chip. 2010, 10, 477-483.

(5) Glavan, A. C.; Christodouleas, D. C.; Mosadegh, B.; Yu, H. D.; Smith, B. S.; Lessing, J.; Fernández-Abedul, M. T., Whitesides, G.M. Folding Analytical Devices for Electrochemical ELISA in Hydrophobic RH Paper. Anal. Chem. 2014, 86 (24), 11999-12007.

(6) Glavan, A. C.; Martinez, R. V.; Maxwell, E. J.; Subramaniam, A. B.; Nunes, R. M. D.; Soh, S.; Whitesides, G. M. Rapid fabrication of pressure-driven open-channel microfluidic devices in omniphobic RF paper. Lab. Chip. 2013, 13, 2922.

(7) Cate, D. M.; Adkins, J. A.; Mettakoonpitak, J., Henry, C. S. Recent Developments in paper-Based Microfluidic Devices. Anal. Chem. 2015, 87 (1), 19-41.

(8) Han, Y. L.; Wang, W.; Hu, J.; Huang, G.; Wang, S.; Lee, W. G.; Lu, T. J.; Xu, F. Benchtop fabrication of three-dimensional reconfigurable microfluidic devices from paper-polymer composite. Lab. Chip. 2013, 13 (24), 4745-4749. 
(9) Noiphung, J.; Songjaroen, T.; Dungchai, W.; Henry, C. S.; Chailapakul, O.; Laiwattanapaisal, W. Electrochemical detection of glucose from whole blood using paper-based microfluidic devices. Anal. Chim. Acta. 2013, 788, 39-45.

(10) Bhakta, S. A.; Borba, R.; Taba Jr, M.; García, C. D; Carrilho, E. Determination of nitrite in saliva using microfluidic paper-based analytical devices. Anal. Chim. Acta. 2014, 809, 117-122.

(11) Chen, G.; Chen, W.; Yen, Y.; Wang, C.; Chang, H.; Chen, C. Detection of Mercury (II) Ions Using Colorimetric Gold Nanoparticles on Paper-Based Analytical Devices. Anal. Chem. 2014, 86, 6843-6849.

(12) Shi, J.; Tang, F.; Xing, H.; Zheng, H.; Bi, L.; Wang, W. Electrochemical Detection of $\mathrm{Pb}$ and $\mathrm{Cd}$ in Paper-Based Microfluidic Devices. J. Braz. Chem. Soc. 2012, 23 (6), 1124-1130.

(13) Dungchai, W.; Chailapakul, O.; Henry, C. S. Electrochemical Detection for Paper-Based Microfluidics. Anal. Chem. 2009, 81, 5821-5826.

(14) Adkins, J.; Boehle, K.; Henry, C. S. Electrochemical paper-based microfluidic devices. Electrophoresis. 2015, 36, 1811-1824.

(15) Rama, E. C.; Costa-García, A.; Fernández-Abedul, M. T. Biosens. Bioelectron. 2017, 88, 34-40.

(16) Rama, E. C.; Costa-García, A.; Fernández-Abedul, M. T. Pin-Based Flow Injection Electroanalysis. Anal. Chem. 2016, 88 (20), 9958-9963.

(17) Nanni, P. I.; González-López, A.; Núñez-Bajo, E.; Madrid, R. E.; Fernández-Abedul, M. T. Staple-Based Electrochemical Platform for Quantitative Analysis on Paper. ChemElectroChem. 2018, 5, 4036-4045.

(18) Yuhi, T.; Nagatani, N.; Endo, T.; Kerman, K.; Takata, M.; Konaka, H.; Namiki, M.; Takamura, Y.; Tamiya, E. Gold nanoparticle based immunochromatography using a resin modified micropipette tip for rapid and simple detection of human chorionic gonadotropin hormone and prostate-specific antigen. Sci. Technol. Adv. Mater. 2006, 7, 276-281.

(19) Yuhi, T.; Nagatani, N.; Endo, T.; Kerman, K.; Takata, M.; Konaka, H.; Namiki, M.; Takamura, Y.; Tamiya, E. Resin-based micropipette tip for immunochromatographic assays in urine samples. $J$. Immunol. Methods. 2006, 312, 54-60.

(20) Bezerra da Silva, R. A.; Rabelo, A. C.; Abarza-Munoz, R. A.; Richter, E. M. Three-Electrode-Integrated Sensor into a Micropipette Tip. Electroanalysis. 2010, 22 (19), 2167-2171.

(21) Zhang, D.; Liu, J.; Nie, J.; Zhou, Y.; Zhang, X. Micropipet Tip-Based Miniaturized Electrochemical Device Combined with Ultramicroelectrode and Its Application in Immobilization-Free Enzyme Biosensor. Anal. Chem. 2013, 85, 2032-2036.
(22) Suarez-Fernandez, A. L., Garcia-Calzón, J. A.; CostaGarcia, A.; Tuñón-Blanco, P. Simple and Reliable Fabrication of Carbon Fiber Ultramicroelectrodes. Electroanalysis. 1991, 3, 413-417.

(23) Abad-Valle, P.; Fernández-Abedul, M. T.; CostaGarcía, A. Genosensor on gold films with enzymatic electrochemical detection of a SARS virus sequence. Biosens. Bioelectron. 2005, 20, 2251-2260.

(24) Wenger, M. D.; DePhillips, P.; Price, C. E.; Bracewell, D. G. An automated microscale chromatographic purification of virus-like particles as a strategy for process development. Biotechnol. Appl. Biochem. 2007, 47, 131-139.

(25) http://www.merckmillipore.com. Last accessed on 2019 , March $19^{\text {th }}$.

(26) Zhang, W.; Chen, Z. Preparation of micropipette tipbased molecularly imprinted monolith for selective micro-solid phase extraction of berberine in plasma and urine samples. Talanta. 2013, 103, 103-109.

(27) Zang, D.; Ge, L.; Yan, M.; Song, X.; Yu, J. Electrochemical immunoassay on a $3 \mathrm{D}$ microfluidic paper-based device. Chem. Commun. 2012, 48, 46834685 .

(28) Bhimji, A.; Zaragoza, A. A.; Live, L. S.; Kelley, S. O. Electrochemical Enzyme-Linked Immunoabsorbent Assay Featuring Proximal Reagent Generation: Detection of Human Immunodeficiency Virus Antibodies in Clinical Samples. Anal. Chem. 2013, 85, 6813-6819.

(29) Sollid, LM; Ludin, K. Diagnosis and treatment of celiac disease. Mucosal Imunol. 2009, 2 (1), 3-7.

(30) Rubio-Tapia, A.; Hill, I. D.; Kelly, C. P.; Calderwood, A. H.; Murray, J. A. ACG Clinical Guidelines: Diagnosis and Management of Celiac Disease. Am. $J$. Gastroenterol. 2013, 108 (5), 656-676.

(31) Rostom, A.; Murray, J. A.; Kagnoff, M. F. American Gastroenterological Association (AGA) Institute Technical Review on the Diagnosis and Management of Celiac Disease. Gastroenterol. 2006, 131 (6), 19812002.

(32) Reif, S.; Lerner, A. Tissue transglutaminase - the key player in celiac disease: a review. Autoimmun. Rev. 2004, 3, 40-45.

(33) Giannetto, M.; Mattarozzi, M.; Umiltá, E.; Manfredi, A.; Quaglia, S.; Careri, M. An amperometric immunosensor for diagnosis of celiac disease based on covalent immobilization of open conformation tissue transglutaminase for determination of anti-tTG antibodies in human serum. Biosens. Bioelectron. 2014, $62,325-330$

(34) Neves, M.M.P.S.; González-García， M.B.; DelerueMatos, C.; Costa-García, A. Multiplexed Electrochemical immunosensor for detection of celiac 
disease serological markers. Sens. Actuators, B. 2013, $187,33-39$

(35) Habtamu, H.B.; Not, T.; De Leo, L.; Longo, S.; Moretto, L.M.; Ugo, P. Electrochemical immunosensor based on nanoelectrode ensembles for the serological analysis of IgG-type tissue transglutaminase. Sensors. 2019, 19, 1233.

(36) Habtamu, H.B.; Sentic, M.; Silvestrini, M.; De Leo, L.; Not, T.; Arbault, S.; Manojlovic, D.; Sojic, N.; Ugo, P. A sensitive electrochemiluminescence immunosensor for celiac disease diagnosis based on nanoelectrode ensembles. Anal. Chem. 2015, 87, 12080-12087.

(37) Cataldo, F.; Montalto, G. Celiac disease in the developing countries: A new and challenging public health problem. World J. Gastroenterol. 2007, 13 (15), 2153-2159.

(38) Macfarlane, S.; Racelis, M.; Muli-Musiime, F. Public health in developing countries. Lancet. 2000, 356, 841846.

(39) Takahashi, K.; Fukada, M.; Kawai, M.; Yokochi, T. Detection of lipopolysaccharide (LPS) and identification of its serotype by an enzyme-linked immunoabsorbent assay (ELISA) using poly-L-lysine. J. Immunol. Methods. 1992, 153, 67-71.

(40) Huang, W.M.; Gibson, S.J.; Facer, P.; Gu, J.; Polak, J.M. Improved section adhesion for immunocytochemistry using high molecular weight polymers of L-Lysine as a slide coating. Histochemistry. 1983, 77, 275-279.

(41) Wilhelm, B.; Meinhardt, A.; Seitz, J. Transglutaminases: purification and activity assays. $J$. Chormatogr. B. 1996, 684, 163-177.

(42) Folk, J.E. Transglutaminases Ann. Rev. Biochem. 1980, 49, 517-531.

(43) Bottari, F.; Moretto, L.M.; Ugo, P. Impedimetric sensing of the immunoenzymatic reaction of gliadin with a collagen-modified electrode. Electrochem. Comm. 2018, 97, 51-55.
(44) Qiao, S.-W.; Piper, J.; Haraldsen, G.; Oynebraten I.; Fleckenstein, B.; Molberg, O.; Chaitan, K.; Sollid, L.M Tissue transglutaminase-mediated formation and cleavage of histamine-gliadin complexes: biological effects and implications for celiac disease. J. Immunol. 2005, 174, 1657-1663.

(45) Dieterich, W.; Esslinger, B.; Trapp, D. Hahn, E.; Huff, T.; Seilmeier, W.; Wieser, H.; Schuppan, D. Cross linking to tissue transglutaminase and collagen favours gliadin toxicity in celiac disease, Gut. 2006, 55, 478484.

(46) Sangha, O.; Catignani, G.L.; Swaisgood, H.E. Characteristics of an immobilized form of transglutaminase: A possible increase in substrate specificity by selective interaction with a protein spacer. J. Agric. Food Chem. 1993, 41, 1337-1342.

(47) Volpe, G.; Compagnone, D.; Draisci, R.; Palleschi, G. $3,3^{\prime}, 5,5^{\prime}$-Tetramethylbenzidine as electrochemical substrate for horseradish peroxidase based enzyme immunoassays. A comparative study. Analyst. 1998, $123,1303-1307$.

(48) Oldfield, L.F.; Bockris J. O’M. Reversible oxidationreduction reactions of aromatic amines. J. Phys. Chem. 1951, 55, 1255-1274.

(49) Diamandis, E. P.; Christopoulos, T. K. Immunoassay; Academic Press: San Diego, 1996, p. 210.

(50) Harding, H.W.J.; Rogers, G.E. Formation of the $\varepsilon-(\gamma-$ glutamyl)lysine cross-link in hair proteins. Investigation of transamidases in hair follicles, Biochemistry. 1972, $11,2858-2863$.

(51) Diamandis, E. P.; Christopoulos, T. K. Immunoassay; Academic Press: San Diego, 1996, p.172.

(52) Gorton, L. A carbon electrode sputtered with palladium and gold for the amperometric determination of hydrogen peroxide, Anal. Chim. Acta 1985, 178, 247253.

\section{For TOC only}

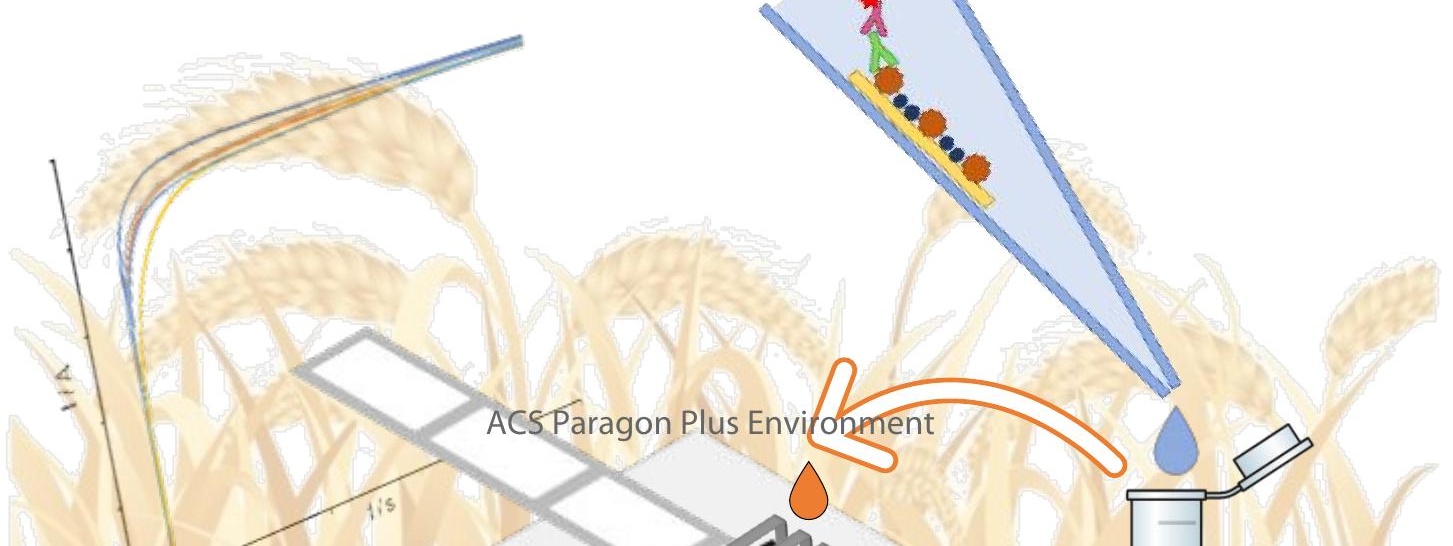

